\title{
Elevation of AMP Levels During Phagocytosis in Acanthamoeba castellanii
}

\author{
By STEVEN W. EDWARDS*† AND FAHMIDA A. DOULAH \\ Department of Microbiology, University College, Newport Road, Cardiff CF2 1TA, U.K.
}

(Received 24 March 1982; revised 16 June 1982)

Addition of particles to growing cultures of the amoeba, Acanthamoeba castellanii resulted in the production of large amounts of AMP, much of which was found extracellularly. At the time of maximal AMP production (13 h growth) adenylate energy charge values in conditioned and fresh medium were 0.58 and $0 \cdot 1$, respectively. Removal of particles from growth medium by filtration lowered the AMP level to $22 \%$ of the control value at the time of maximal AMP production; addition of particles to conditioned medium raised the level of AMP 3.6-fold.

Uptake of killed yeast was accompanied by relatively small changes in levels of ATP and ADP, but large $(5 \cdot 7$-fold) changes in AMP levels. Successive cycles of phagocytosis were paralleled by cyclic changes in AMP levels.

\section{INTRODUCTION}

The soil amoeba Acanthamoeba castellanii, in common with other free-living amoebae (Chapman-Andresen, 1977), relies entirely on processes of endocytosis for uptake of nutrients. The plasma membrane of this organism has many similarities in its macromolecular composition to mammalian cells (Korn \& Wright, 1973) and indeed the endocytic mechanisms appear similar to those occurring in many types of mammalian cells (Silverstein et al., 1977; Klebanoff \& Clark, 1978). Apart from feeding mechanisms, endocytosis is also important for the microbiocidal action of certain white blood cells (Klebanoff \& Clark, 1978), membrane internalization and re-cycling (Schneider et al., 1979; Bowers, 1980; Bowers et al., 1981) and other important cellular functions (Silverstein et al., 1977). While in $A$. castellanii pinocytosis (fluid uptake) appears to be a continuous feeding process (Bowers \& Olszewski, 1972), phagocytosis (particle uptake) can be induced by the addition of latex beads (Weisman \& Korn, 1967; Wetzel \& Korn, 1969), yeasts (Ryter \& Bowers, 1976) or horse red blood cells (Brown et al., 1975).

During exponential growth of $A$. castellanii in batch culture in a glucose/proteose peptone/yeast extract medium, the initial $30 \mathrm{~h}$ are unusual in several respects (Edwards \& Lloyd, $1977 a, b)$. Respiration rates are low, but upon the addition of $1 \mathrm{~mm}$-cyanide may be stimulated by up to $50 \%$ (Edwards \& Lloyd, 1977b); glucose disappearance is negligible and adenylate energy charge values are low (less than $0 \cdot 1$ ) mainly due to the production of large amounts of AMP, much of which is found extracellularly (Edwards \& Lloyd, 1977a). When grown in conditioned medium (i.e. having previously supported growth for $30 \mathrm{~h}$ ), respiration is still cyanide-stimulated (Edwards \& Lloyd, 1977b), but glucose disappearance is detectable from time zero, and low levels of extracellular AMP are found (Edwards \& Lloyd, 1977a). Thus, it was suggested that the particles that are produced after autoclaving growth medium are preferentially ingested by the amoebae and that this phagocytic process is responsible for the elevated AMP levels.

$\dagger$ Present address: Department of Medical Biochemistry, Welsh National School of Medicine, Heath Park, Cardiff CF4 4 XN, U.K. 
In the present study we have further examined this phenomenon using various modifications of the growth medium and by feeding the cells with heat-killed yeasts. We show that phagocytosis of particles of medium or yeast results in the production of large amounts of AMP, much of which is found extracellularly.

\section{METHODS}

Growth of the organism. Acanthamoeba castellanii was maintained and grown with shaking at $30{ }^{\circ} \mathrm{C}$ exactly as described previously (Edwards et al., 1977) in a medium containing $(\%, w / v)$ : Difco proteose peptone, $0.75 ;$ Oxoid yeast extract, $0 \cdot 75$; glucose, $1 \cdot 5$. Organisms were counted in a Fuchs-Rosenthal haemocytometer slide (Baird \& Tatlock, Chadwell Heath, Romford Essex) after suitable dilution with $50 \mathrm{~mm}-\mathrm{MgCl}_{2}, \mathrm{pH} 7 \cdot 4$.

Measurements of phagocytosis. Dried Saccharomyces cerevisiae $(0.5 \mathrm{~g})$ was heated to $100{ }^{\circ} \mathrm{C}$ for $1 \mathrm{~h}$ in $10 \mathrm{ml}$ of $50 \mathrm{~mm}$-phosphate buffer, $\mathrm{pH} 7 \cdot 4$ (Jenson \& Bainton, 1973). The suspension was centrifuged at $1000 \mathrm{~g}$ for $5 \mathrm{~min}$, the supernatant discarded and the pellet washed twice in 5 vol. of the above buffer. The heat-killed organisms were kept as a thick slurry at $4{ }^{\circ} \mathrm{C}$ for 1 week until required.

For measurements of phagocytosis, amoebae were grown for $36 \mathrm{~h}$ (cell density of approximately $10^{6}$ cells $\mathrm{ml}^{-1}$ ) in duplicate flasks. The medium from one culture was collected by centrifugation at $1000 \mathrm{~g}$ for $10 \mathrm{~min}$ at $30^{\circ} \mathrm{C}$ and the supernatant was termed 'conditioned medium'. This was used to dilute the contents of the second flask to a cell density of approximately $2 \times 10^{5}$ cells $\mathrm{ml}^{-1}$; the flask was equilibrated at $30^{\circ} \mathrm{C}$ with shaking for 30 min prior to the addition of the heat-killed yeast. Initiation of phagocytosis was by the addition of the killed yeast suspension (usually about $10-35 \mu \mathrm{l}$ to $50 \mathrm{ml}$ culture) and samples were removed at frequent intervals for cell counts, or measurements of adenine nucleotides.

Experiments with conditioned medium. Conditioned medium was prepared as described above. Particles formed after autoclaving were removed by filtration through $0.22 \mu \mathrm{m}$ pore-size filters. Where indicated, particles were introduced into conditioned media by washing the filter. Thus, four types of growth media could be produced: (1) freshly-autoclaved media; (2) freshly-autoclaved, filtered media; (3) conditioned media and (4) conditioned media with the particles from (2) added to it. All media were autoclaved before inoculation $\left(10^{5} \mathrm{cells} \mathrm{ml}^{-1}\right)$ and amoebae were allowed to grow for about $24 \mathrm{~h}$.

Analytical methods. Measurements of adenine nucleotides were as described previously (Edwards et al., 1975) using luciferin-luciferase assays on $1 \mathrm{ml}$ of culture prepared by extraction with $0.3 \mathrm{ml}$ boiling chloroform. Rapid mixing using a 'Whirlimixer' ensured quenching within 0.2 s. ATP, ADP and AMP were measured following the procedure of Chapman et al. (1971) using ATP as calibration standard. After filtration of the culture (Whatman GF/A filters), adenine nucleotides in the growth medium were measured using $1 \mathrm{ml}$ of the filtrate. The yeast suspension contained only AMP at a concentration of $0.26 \mathrm{nmol} \mathrm{ml}^{-1}$ and this value was subtracted from all AMP measurements. Oxygen uptake was measured at $30^{\circ} \mathrm{C}$ using the 'open' system of Degn \& Wohlrab (1971). The system consisted of a stainless steel reaction vessel (working volume $4.5 \mathrm{ml}$ ) fitted with a membrane-covered Clark electrode (type D602; Radiometer, Copenhagen). With a constant partial pressure of oxygen in the gas phase, the respiration rate $\left(V_{\mathrm{r}}\right)$ was calculated after the attainment of a steady-state oxygen concentration in the liquid phase by the equation: $V_{\mathrm{r}}=K\left(T_{\mathrm{G}}-T_{\mathrm{L}}\right)$, where $K$ is the oxygen transfer constant, $T_{\mathrm{G}}$ is the oxygen concentration in the gas phase and $T_{\mathrm{L}}$ is the oxygen concentration in the liquid phase.

\section{RESULTS}

\section{Changes in AMP concentration during growth}

Previous work had suggested that the large quantities of AMP produced during the initial phase of growth in fresh medium may be the result of phagocytosis of particles that are produced after autoclaving (Edwards \& Lloyd, 1977a). These high levels of AMP were not apparent during growth in conditioned medium which did not contain particles. Differing growth conditions, as described in Methods, were employed to further test this hypothesis. In culture 1 (freshly autoclaved medium) the AMP concentration was highest after about $15 \mathrm{~h}$ growth, at approximately $100 \mathrm{nmol}$ (ml culture) $)^{-1}$ (Fig. 1 a). In culture 2 (freshly autoclaved but filtered) the AMP concentration was reduced to about $22 \mathrm{nmol}(\mathrm{ml} \text { culture })^{-1}$. In culture 3 (conditioned media containing no particles) AMP reached a maximum concentration of about $5 \mathrm{nmol}(\mathrm{ml}$

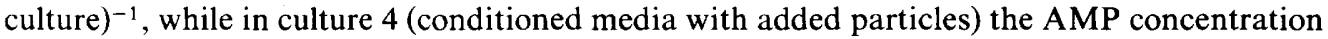
reached about $18 \mathrm{nmol}$ (ml culture $)^{-1}$. Thus, removal of particles from fresh media reduced the AMP concentration to about $22 \%$ of the control value, whereas addition of particles to conditioned media elevated the AMP concentration 3.6-fold. Corresponding adenylate energy charge values for conditioned medium and fresh medium at $13 \mathrm{~h}$ growth were 0.58 and $0 \cdot 1$, respectively (Fig. $1 b$ ). 


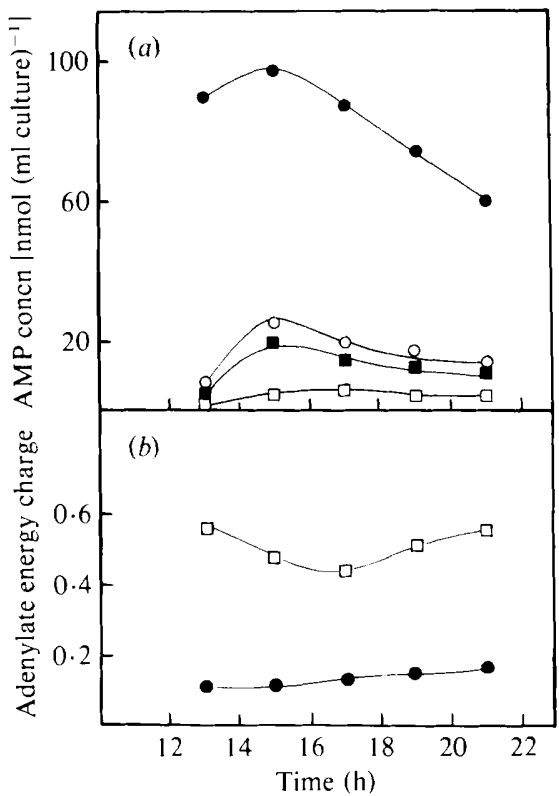

Fig. 1. Changes in AMP and adenylate energy charge values during growth of $A$. castellanii. Organisms were inoculated $\left(10^{5}\right.$ cells $\left.\mathrm{ml}^{-1}\right)$ at time zero into: freshly autoclaved medium $(O)$; freshly autoclaved, filtered medium $(O)$; conditioned medium $(\square)$ and conditioned medium with added particles $(\boldsymbol{\square})$. Samples were removed at intervals for cell counts (data not shown) and measurement of $(a)$ AMP and (b) adenylate energy charge, defined by Atkinson \& Walton (1967) as: adenylate energy charge $=($ ATP $\left.+\frac{1}{2} \mathrm{ADP}\right) /(\mathrm{ATP}+\mathrm{ADP}+\mathrm{AMP})$.

\section{Phagocytosis of yeast}

While the above evidence showed that the presence or absence of particles in the growth media greatly altered AMP levels, a more direct, controllable system of phagocytosis was tested. Heat-killed yeasts were added to a suspension of amoebae and the rate of phagocytosis was measured by counting the number of yeasts free in the medium (Fig. 2). With an initial ratio of 10 yeasts to 1 amoeba phagocytosis was completed by $30 \mathrm{~min}$, i.e. virtually no yeast were detected free in the medium. When the initial ratio of yeast to amoebae was increased to $20: 1$, the rate of phagocytosis was biphasic. By 4 min the amoebae had engulfed approximately 16 yeasts each; no further phagocytosis took place for a further 6 min and then another burst of phagocytosis was observed. Presumably this lag phase represented a period of digestion of phagocytosed yeasts which must be completed before further uptake could occur. When the initial ratio of yeast to amoeba was increased further to $40: 1$, each amoeba took up approximately 30 yeast during the initial burst of phagocytosis. This increased uptake presumably results from the increased collision of yeasts with amoebae, which must occur before ingestion; however, it must be pointed out that we have not distinguished between yeasts which were engulfed and those merely attached to the cell surface. Also, at such high yeast to amoeba ratios extensive clumping of amoebae occurs, probably trapping yeasts. Thus, these numbers of yeast ingested per cell must be considered to be maximal.

\section{Changes in oxygen uptake rates during phagocytosis of yeasts}

A suspension of amoebae $\left(2 \times 10^{5}{\left.\text { cells } \mathrm{ml}^{-1}\right)}^{-}\right.$in conditioned medium was placed in the open oxygen electrode vessel and allowed to reach a steady-state with air in the gas phase. The steadystate respiration was calculated to be $1.54 \mathrm{nmol} \mathrm{O}_{2} \mathrm{~min}^{-1}$ (ml culture) ${ }^{-1}$ (Fig. 3). As indicated, yeasts were added to give a yeast to amoeba ratio of $10: 1$ and, after a lag phase of about $15 \mathrm{~min}$, the steady-state level of oxygen dropped indicating an increase in respiration rate to $1.88 \mathrm{nmol} \mathrm{O}_{2} \min ^{-1}$ ( $\mathrm{ml}$ culture $)^{-1}$. Thus, a $22 \%$ increase in respiration rate occurred during phagocytosis. 


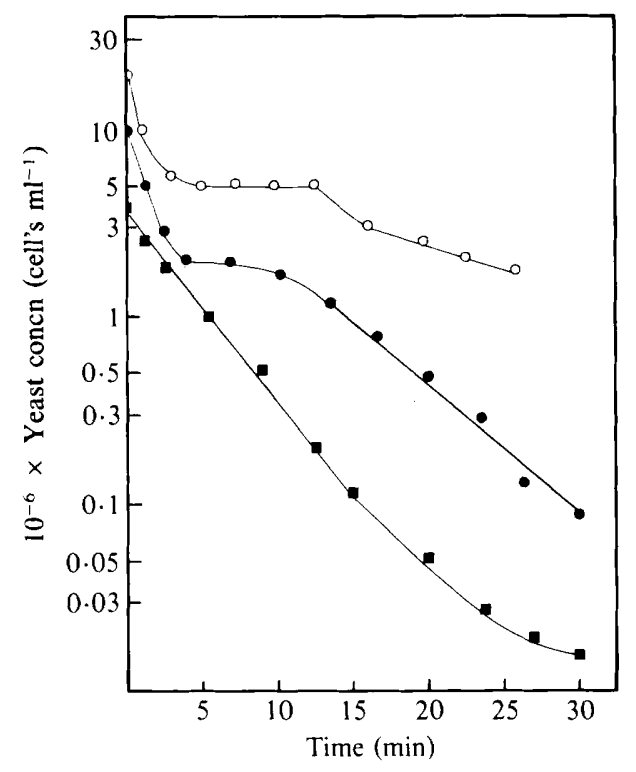

Fig. 2. Phagocytosis of yeast by A castellanii. Saccharomyces cerevisiae was heat-killed as described in Methods. Cultures of $A$. castellanii were grown to approximately $10^{6}$ cells $\mathrm{ml}^{-1}$ and diluted to $4 \times 10^{5}$ cells $\mathrm{ml}^{-1}(\square)$ or $5 \times 10^{5}$ cells $\mathrm{ml}^{-1}(\boldsymbol{O}, \bigcirc)$ with conditioned medium. At time zero yeast were added to give initial yeast to amoeba ratios of $40: 1(\bigcirc) ; 20: 1(\mathcal{O})$ and $10: 1(\boldsymbol{\square})$. Phagocytosis was estimated by microscopic counts of the number of yeast free in the medium.

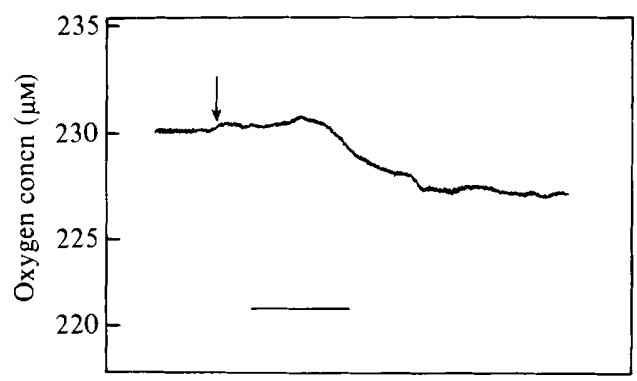

Fig. 3. Changes in oxygen uptake rates during phagocytosis of yeasts. A culture of A. castellanii was grown to $10^{6}$ cells $\mathrm{ml}^{-1}$ and diluted to $2 \times 10^{5}$ cells $\mathrm{ml}^{-1}$ with conditioned medium. Diluted culture $(4.5 \mathrm{ml})$ was introduced into the open oxygen electrode system until the steady-state concentration of oxygen in the liquid phase was attained. Heat-killed yeasts were added (arrow) to give an initial ratio of yeast to amoeba of $10: 1$. The bar marker represents $10 \mathrm{~min}$.

\section{Changes in adenine nucleotide pool levels during phagocytosis of yeast}

Yeast were added to a suspension of amoebae to give an initial ratio of 30 yeast to 1 amoeba. Phagocytosis was initially rapid and proceeded in three distinct phases (Fig. $4 a$ ). The first phase was completed by about $20 \mathrm{~min}$ and the second by about $45 \mathrm{~min}$. Concentrations of ATP (Fig. $4 b$ ), ADP (Fig. 4c) and AMP (Fig. 4d) were constant before the addition of yeast and were about $0.6,1.55$ and $6 \mathrm{nmol}(\mathrm{ml} \text { culture })^{-1}$, respectively, where the culture included cells plus medium. Upon the addition of yeast, levels of all three adenine nucleotides dropped initially; ATP levels dropped by $50 \%$, ADP by $26 \%$ and AMP by $81 \%$. Levels of all three then increased as the initial burst of phagocytosis declined, decreased again (AMP remained constant) at the onset of the second burst of phagocytosis and then declined as the second burst of phagocytosis slowed down. The third phase of phagocytosis did not show such dramatic changes in adenine nucleotide levels. The maximal increases in levels (about $45 \mathrm{~min}$ after the addition of yeast) 


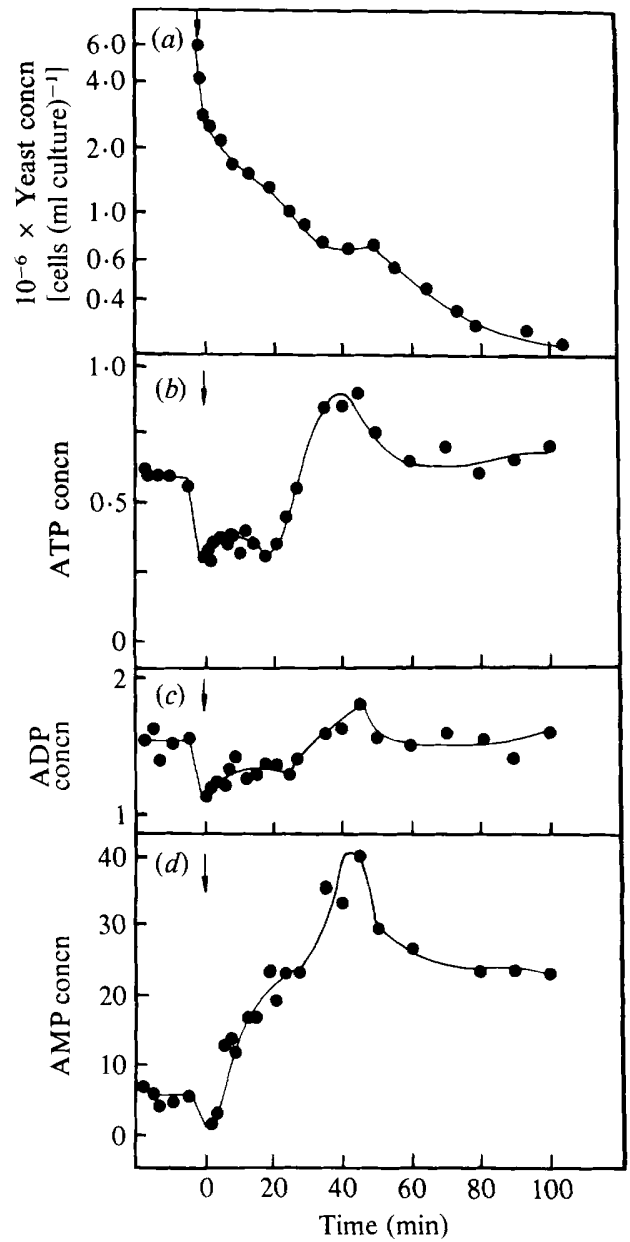

Fig. 4. Changes in adenine nucleotide pool levels during phagocytosis of yeasts. A culture of $A$. castellanii was grown to $10^{6}$ cells $\mathrm{ml}^{-1}$ and diluted to $1.8 \times 10^{5}$ cells $\mathrm{ml}^{-1}$ with conditioned medium. The culture was returned to the growth vessel for $30 \mathrm{~min}$. Five samples were removed from the culture and rapidly extracted with boiling chloroform, prior to the addition of yeasts. At time zero (arrow) heatkilled yeasts were added to give an initial yeast to amoeba ratio of $32: 1$. At frequent time intervals, samples were removed for measurement of (a) yeast cell numbers, (b) ATP, (c) ADP and (d) AMP. Levels of adenine nucleotides are expressed as $\mathrm{nmol}(\mathrm{ml} \text { culture })^{-1}$, where culture included cells plus medium. Results are typical of five separate experiments.

above those before phagocytosis was initiated were: ATP 50\%; ADP $16 \%$; AMP $567 \%$. At this time no ATP could be detected in the culture filtrate, while $4 \%$ of the ADP and over $40 \%$ of the AMP were detected extracellularly.

\section{DISCUSSION}

The results presented here confirm the previous suggestion (Edwards \& Lloyd, 1977a) that phagocytosis of particles by $A$. castellanii results in elevation of AMP levels, thus lowering values of adenylate energy charge. This phenomenon therefore provides another example which, together with some cell cycle-dependent variations (Lloyd et al., 1982), argues against the concept of stabilized adenylate energy charge values in growing organisms (Chapman \& Atkinson, 1977). Phagocytosis has been observed under two conditions in the present study using particles of growth medium that are produced upon autoclaving the medium and a more controllable experimental system using heat-killed yeast. Removal of particles from the growth 
medium reduced AMP levels to $22 \%$ of the maximal value, while addition of these particles to conditioned medium elevated AMP levels 3.6-fold. Phagocytosis of yeasts showed that elevation of AMP levels occurred within 3 min of the initiation of uptake. Repeating cycles of AMP production corresponding to successive cycles of uptake of yeasts add further strength to these conclusions.

Whether AMP production and subsequent extracellular export is a primary or secondary response to phagocytosis is not known at present. While all three adenine nucleotides showed responses similar to that of AMP during yeast uptake, the magnitude of the changes in ATP and ADP were less dramatic. Final levels of ATP were only about $15 \%$ higher than the level before the initiation of phagocytosis, whereas AMP levels were increased by a maximum of $5 \cdot 7$-fold. Since ATP is believed to be necessary for the phagocytic response (Silverstein et al., 1977), a drop in ATP levels at the onset of yeast uptake does not seem surprising. ATP turnover in growing micro-organisms is very rapid (Chapman \& Atkinson, 1977) and, since A. castellanii were not nutrient-limited, regeneration of ATP, with perhaps an 'overshoot' in production, rapidly occurred. Changes in AMP levels may be correlated with this phenomenon, since ADP produced by ATP hydrolysis may be further dephosphorylated to AMP and exported from the cells. In this way favourable ATP : ADP or ATP : AMP ratios may be maintained intracellularly (Knowles, 1977). Alternatively, there may also be some contribution to elevated AMP levels by increased RNA degradation (Klebanoff \& Clark, 1978).

The increased oxygen uptake rate which was observed during phagocytosis resembles the 'metabolic burst' of leucocytes (see Silverstein et al., 1977; Klebanoff \& Clark, 1978 for references), where phagocytosing cells may show a two- to threefold increase in oxygen consumption rate. It is doubtful, however, in some cases if this cyanide-insensitive respiration is directly concerned with the increased energy demands. The response in $A$. castellanii, apart from being of much lower magnitude, was also different in that the increase in oxygen uptake rate occurred much later after the initiation of phagocytosis than can be considered typical for a 'metabolic burst.'

Further work is necessary to ascertain the origin and fate of the extracellular AMP and to determine any similarities and/or differences in endocytic properties to those in mammalian cells.

During the course of this work S. W.E. held a University of Wales Postdoctoral Fellowship.

\section{REFERENCES}

Atkinson, D. E. \& Walton, G. M. (1967). Adenosine triphosphate conservation in metabolic regulation. Journal of Biological Chemistry 242, 3239-3241.

BOWERS, B. (1980). A morphological study of plasma and phagosome membranes during endocytosis in Acanthamoeba. Journal of Cell Biology 84, 246-260.

Bowers, B. \& OlszewsKI, T. E. (1972). Pinocytosis in Acanthamoeba castellanii. Kinetics and morphology. Journal of Cell Biology 53, 681-694.

Bowers, B., Olszewski, T. E. \& Hyde, J. (1981). Morphometric analysis of volumes and surface areas in membrane compartments during endocytosis in Acanthamoeba. Journal of Cell Biology 88, 509-515.

Brown, R. C., Bass, H. \& Coombs, J. P. (1975). Carbohydrate binding proteins involved in phagocytosis by Acanthamoeba. Nature, London 254, 434-435.

Chapman, A. G. \& AtKinson, D. E. (1977). Adenine nucleotide concentrations and turnover rates. Correlation with biological activity in bacteria and yeast. Advances in Microbial Physiology 15, 253-306.

Chapman, A. G., Fall, L. \& AtKinson, D. E. (1971). Adenylate energy charge in Escherichia coli during growth and starvation. Journal of Bacteriology 108, 1072-1086.
Chapman-Andresen, C. (1977). Endocytosis in freshwater amoebas. Physiological Reviews 57, 371385.

DegN, H. \& Wohlrab, H. (1971). Measurement of steady-state values of respiration rate and oxidation levels of respiratory pigments at low oxygen tensions. A new technique. Biochimica et biophysica acta 245, 347-355.

EDWards, S. W. \& Lloyd, D. (1977a). Changes in oxygen uptake rates, enzyme activities, cytochrome amounts and adenine nucleotide pool levels during growth of Acanthamoeba castellanii in batch culture. Journal of General Microbiology 102, 135-144.

EDWards, S. W. \& Lloyd, D. (1977b). Cyanideinsensitive respiration in Acanthamoeba castellanii. Changes in sensitivity of whole cell respiration during exponential growth. Journal of General Microbiology 102, 207-213.

Edwards, C., Statham, M. \& Lloyd, D. (1975). The preparation of large-scale synchronous cultures of the trypanosomatid, Crithidia fasciculata by cell-size selection: changes in respiration and adenylate charge through the cell cycle. Journal of General Microbiology 88, 141-152. 
Edwards, S. W., Chagla, A. H., Griffiths, A. J. \& Lloyd, D. (1977). The cytochromes of Acanthamoeba castellanii. Biochemical Journal 168, 113-121.

JENSEN, M. S. \& BaINTON, D. F. (1973). Temporal changes in the $\mathrm{pH}$ within the phagocytic vacuole of the polymorphonuclear neutrophilic leukocyte. Journal of Cell Biology 56, 379-388.

Klebanoff, S. J. \& Clark, R. A. (1978). The Neutrophil: Function and Clinical Disorders. Amsterdam: North-Holland Publishing Co.

KNOWLES, C. J. (1977). Microbial regulation by adenine nucleotide pools. Symposia of the Society for General Microbiology 27, 241-283.

KorN, E. D. \& Wright, P. L. (1973). Macromolecular composition of an amoeba plasma membrane. Journal of Biological Chemistry 248, 439-447.

Lloyd, D., PoOle, R. K. \& EdwaRds, S. W. (1982). In The Cell Division Cycle: Temporal Organization and Control of Cellular Growth and Reproduction, pp.265294. London \& New York : Academic Press.
RYTER, A. \& Bowers, B. (1976). Localization of acid phosphatase in Acanthamoeba castellanii with light and electron microscopy during growth and after phagocytosis. Journal of Ultrastructure Research 57. 309-321.

Schneider, Y-J., Tulkens, P., DE Duve, C. \& Trouet, A. (1979). Fate of plasma membrane during endocytosis. II. Evidence for recycling (shuttle) of plasma membrane constituents. Journal of Cell Biology 82, 466-474.

Silverstein, S. C., Steinman, R. M. \& Cohn, Z. A. (1977). Endocytosis. Annual Reviews of Biochemistry 46, 669-722.

WeIsman, R. A. \& KoRn, E. D. (1967). Phagocytosis of latex beads by Acanthamoeba castellanii I. Biochemical properties. Biochemistry 6, 485-497.

WETZEL, M. G. \& KORN, E. D. (1969). Phagocytosis of latex beads by Acanthamoeba castellanii (Neff). III. Isolation of the phagocytic vesicles and their membranes. Journal of Cell Biology 43, 90-104. 